Review Article

\title{
MicroRNA as a Potential Biomarker and Treatment Strategy for Ischemia-Reperfusion Injury
}

\author{
Mingming Cao $\mathbb{D},{ }^{1}$ Wenjing Song $\mathbb{D},{ }^{1}$ Runyu Liang $\mathbb{D}^{1},{ }^{1}$ Lili Teng $\mathbb{D},{ }^{1}$ Mei Zhang $\mathbb{D},^{1}$ \\ Jiyao Zhang ${ }^{1},{ }^{1}$ and Luwen Zhu iD ${ }^{2}$ \\ ${ }^{1}$ Heilongjiang University of Chinese Medicine, 24 Heping Road, Xiangfang District, Heilongjiang, Harbin 150040, China \\ ${ }^{2}$ Second Affiliated Hospital of Heilongjiang University of Chinese Medicine, 411 Guogeli Street, Nangang District, Heilongjiang, \\ Harbin 150001, China
}

Correspondence should be addressed to Luwen Zhu; zhuluwen1983@126.com

Received 23 May 2021; Accepted 2 November 2021; Published 20 November 2021

Academic Editor: Wilfred van IJcken

Copyright (c) 2021 Mingming Cao et al. This is an open access article distributed under the Creative Commons Attribution License, which permits unrestricted use, distribution, and reproduction in any medium, provided the original work is properly cited.

\begin{abstract}
Ischemia-reperfusion (I/R) injury is a progressive injury that aggravates the pathological state when the organ tissue restores blood supply after a certain period of ischemia, including the myocardial, brain, liver, kidney, and intestinal. With growing evidence that microRNAs (miRNAs) play an important role as posttranscription gene silencing mediators in many I/R injury, in this review, we highlight the microRNAs that are related to I/R injury and their regulatory molecular pathways. In addition, we discussed the potential role of miRNA as a biomarker and its role as a target in I/R injury treatment. Developing miRNAs are not without its challenges, but prudent design combined with existing clinical treatments will result in more effective therapies for I/R injury. This review is aimed at providing new research results obtained in this research field. It is hoped that new research on this topic will not only generate new insights into the pathophysiology of miRNA in I/R injury but also can provide a basis for the clinical application of miRNA in I/R.
\end{abstract}

\section{Introduction}

Mature microRNA (miRNA) is a small but noncoding single-stranded RNA molecule with mature transcripts of 18-25 nucleotides. It acts as a negative regulator of gene expression by combining with the $3^{\prime}$-untranslated region (UTR) of complementary or partially complementary target messenger RNAs (mRNAs) to inhibit or degrade its expression. As a new gene expression regulation mechanism, its importance as a noncoding regulatory RNA is extensively studied [1]. miRNAs can participate in a variety of biological processes, such as gene transcription, posttranscriptional processing, cell differentiation, ontogeny, heredity, and epigenetics [2]. Notably, key roles have emerged in pathophysiological fields such as ischemiareperfusion (I/R) injury [3].

\section{2. miRNA Biosynthesis and Function}

miRNAs are believed to be transcribed from DNA, and although it cannot be translated, it can regulate gene expression by changing the translation efficiency and/or stability of targeted mRNAs after transcription [4]. The initial transcript (primary miRNA; pri-miRNA) of miRNA is a long RNA transcript containing at least one hairpin-shaped miRNA precursor, which has a short existence time. It is cut by Ribonuclease III (RNase III) endonuclease and/or splicing elements similar to Drosha to form a 60-70 nt premiRNA with stem ring structure [5]. This structure is then transported from the nucleolus into the cytoplasm by Exportin-5, a guanosine triphosphate- (GTP-) bound form(RanGTP-) dependent double-stranded RNA (dsRNA) binding protein that mediates the nuclear output of miRNAs 
[6]. In the cytoplasm, Dicer-like endonuclease cuts premiRNA into a double-stranded 18 25 nt mature miRNA and unwinds the chain into strand of miRNA and a miRNA passenger chain $(\operatorname{miRNA} *)$. In one instance, the miRNA chains combine with the RNA-induced silencing complex (RISC), whereas the miRNA* is subsequently degraded. The miRNA/RISC complex combines with specific target sequence mRNA in a partially complementary manner, especially through base pairing in the $3^{\prime}$-UTR of mRNA, which leads to mRNA transcription inhibition or elimination, thus inhibiting protein expression [7].

miRNAs can regulate gene expression, depending on their ability to complement one or more mRNA partial sequences, which are generally in the $3^{\prime}$-UTR region. This binding of miRNAs to target mRNAs can inhibit protein translation. In some cases, dsRNA is formed by binding to miRNAs, thus triggering the degradation of mRNAs [8]. However, in other cases, miRNA complexes only inhibit protein translation machines but can also inhibit protein translation by other methods that do not degrade RNA [9]. Most miRNAs can be found in the cytoplasm. However, increasing evidence shows that some mature miRNAs can reenter the nucleus and regulate the biological occurrence of other miRNAs or even their own expression [10]. The mediated nuclear transport of mature miRNAs is also dependent on the association of importin-8 (IPO8) with the argonaute-2 (Ago2) complex which determines the RISC-carrying miRNA chain transport to the nucleus [11]. In addition, the exogenous gene miRNAs regulate the expression of target genes, establish cell-to-cell communication, and participate in regulating different biological processes. Furthermore, exon-shuttle miRNAs and other RNAs are transported into cells without being degraded by extracellular RNAs. Montecalvo et al. [12] found that the transfer of nano-ovum $(<100 \mathrm{~nm})$ obtained from the intracellular pathway is a new communication mechanism between dendritic cells, while the transfer mechanism of exogenous shuttle miRNAs between dendritic cells serves a role in communication and posttranscriptional regulation (Figure 1).

Because the function of most miRNA inhibitory genes depends on partial complementation, a single miRNA can target multiple mRNA, while multiple miRNAs can also act on a single mRNA, thus cooperatively regulating the expression intensity of genes in different tissues and cells. Therefore, miRNAs may have extensive fine-tuning effects on protein-coding genes. Undoubtedly, the discovery of miRNAs has changed our understanding of gene regulation in the postgenome era.

\section{3. miRNAs and I/R Injury}

$\mathrm{I} / \mathrm{R}$ is a pathological state characterized by reduced tissue blood supply, accompanied by adverse reactions such as metabolite accumulation, vascular permeability [13], interstitial edema [14], and endothelial dysfunction [15] during ischemia, followed by the recovery of perfusion and accompanied reoxygenation, which increases the sensitivity of tis- sues in ischemia-induced hypoxia to oxygen after recovery of circulation, resulting in the activation of inflammatory factors and excessive autophagy. Furthermore, increases in reactive oxygen species (ROS), endothelial injury, and complement activation result in secondary injury to ischemic tissue and I/R injury [16]. I/R injury leads to some problems, including acute myocardial infarction (AMI) [17], ischemic stroke (IS) [18], acute kidney injury (AKI) [19], trauma [20], circulatory arrest [21], sickle cell disease, and sleep apnea [22]. In addition, I/R injury is a major problem in organ transplantation and general surgery [23]. Some evidences show that miRNAs are involved in the regulation of many pathophysiological processes of I/R injury with significant differential expression; these small RNAs are becoming promising therapeutic targets for ischemic diseases (Figure 2).

3.1. Myocardial Ischemia/Reperfusion Injury. Early reperfusion after AMI is an effective treatment method, but which may lead to myocardial cell dysfunction [24]. Therefore, it is prudent to reveal new molecular targets and networks involved in triggering and resisting myocardial I/R injury pathophysiology. Some studies have suggested that miRNAs are involved in this process. Dong et al. [25] used microarray analysis to investigate the expression characteristics of miRNAs in rat hearts after $6 \mathrm{~h}$ of I/R in early AMI. The results showed that among the 341 mature miRNA sequences, 38 miRNAs were differentially expressed in infarcted regions (21 up and 17 downregulated), and 33 miRNAs were abnormally expressed in marginal regions compared with noninfarcted regions. Specifically, miR-21, which is present in cardiac progenitor cell-derived exosomes, is significantly downregulated in infarcted areas and upregulated in border areas. Previously, we used denovirus-mediated miR-21 gene transfer technology, further confirmed the protective effect of miR-21 on myocardial cell injury caused by ischemia [26]. Overexpression of miR-21 significantly reduced the necrotic area of the heart after I/R and reduced the necrotic volume of the myocardium, thus leading to long-term remodeling of the heart and reduction of apoptosis. This was the first time that multiple miRNAs were confirmed to have abnormal expression in the early $(6 \mathrm{~h})$ border and infarcted area of AMI. However, it is still necessary to reveal the complex role of miRNAs in the pathophysiological process of AMI through computational analysis, prediction, or depletion experiments.

The fatty acid synthase (Fas) death pathway is considered an important medium for myocardial cell death and AMI during myocardial ischemia reperfusion injury (MI/ RI) $[27,28]$. The latest research by $\mathrm{He}$ et al. [29] used microarray analysis technology to show miRNAs differentially expressed after morphine preconditioning treatment. The results show that overexpression of $\mathrm{miR}-133 \mathrm{~b}-5 \mathrm{p}$ increased cell activity, inhibited lactate dehydrogenase activity, and inhibited Fas gene and protein expression, thus reducing cell apoptosis and alleviating hypoxia/reoxygenation (H/R) intrusion. However, the in vitro data provided by $\mathrm{miR}$ 133b-5p mimetics and inhibitors may not fully replicate the in vivo environment and cannot fully prove that miR- 


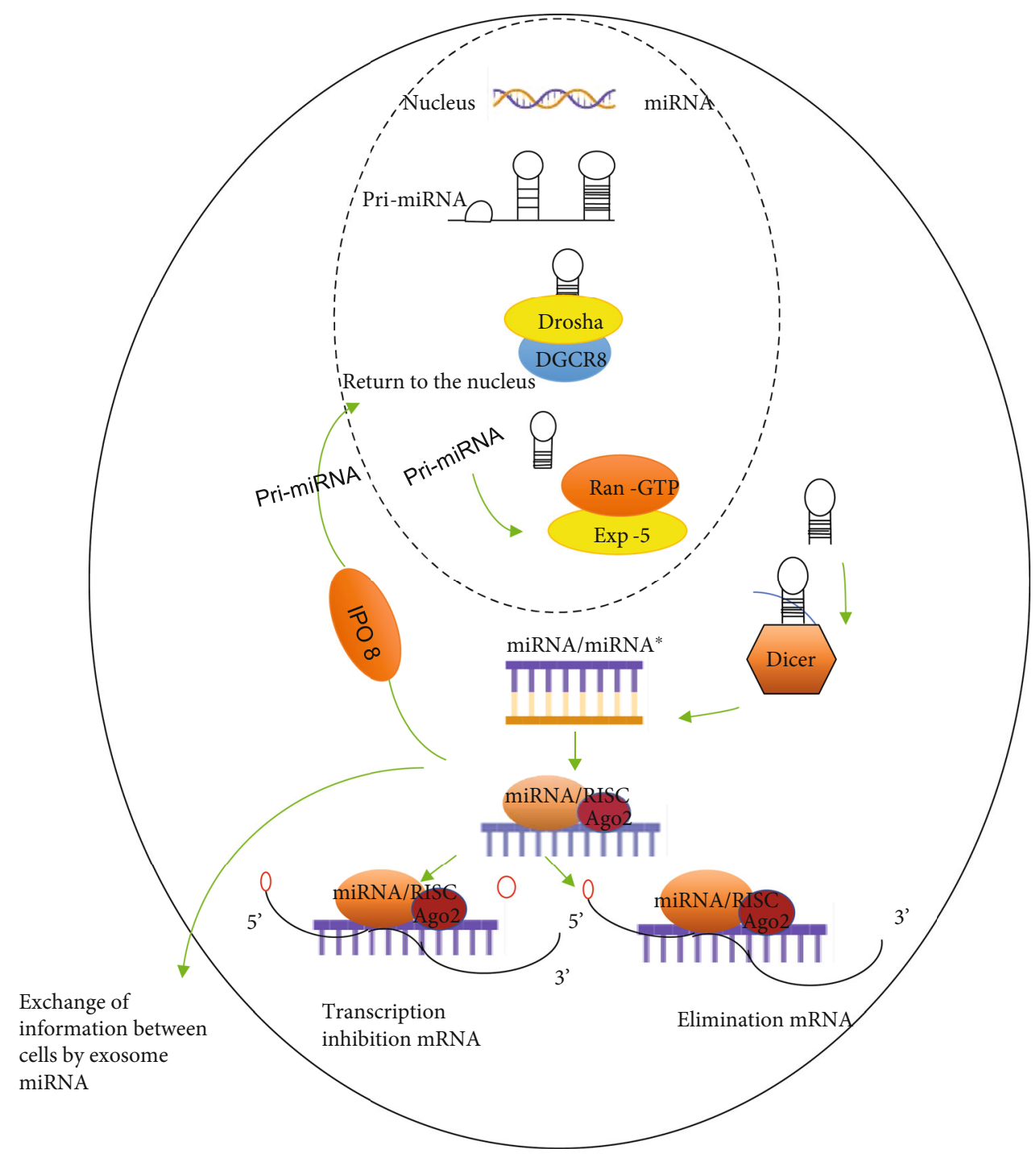

FIGURE 1: miRNA biosynthesis processing.

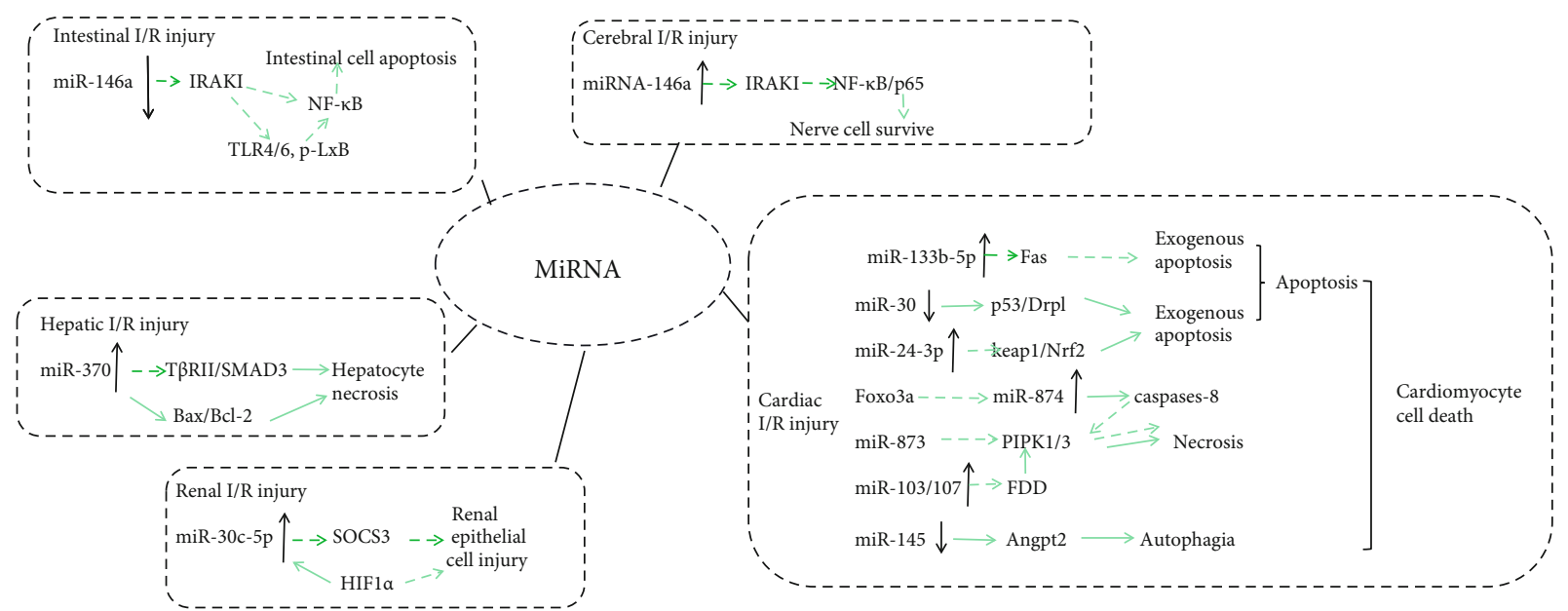

FIGURE 2: miRNAs in cerebral, intestinal, hepatic, cardiac, and renal I/R injury. Solid arrow indicates promote. Dotted arrow indicates inhibit. 
$133 \mathrm{~b}-5 \mathrm{p} / \mathrm{Fas}$ plays a key role in cardiac protection against I/ $\mathrm{R}$ injury mediated by morphine pretreatment. Simultaneously, miR-133b-5 may also have a "one-to-man" target gene regulation mechanism, and further relevant pathways should therefore be explored.

3.2. Brain Ischemia/Reperfusion Injury. Rapid reconstruction of occluded blood vessels and early reperfusion to limit cerebral ischemia injury is an important stroke treatment strategy $[18,30]$. However, this approach also leads to $I / R$ injury. In recent years, the expression of miRNAs in the brain tissues has received considerable attention. The basic biological research process of miRNAs in IS has been rapidly carried out and is expected to be upgraded. Min et al. [1] found that miRNA expression profiles changed significantly, with 15 miRNAs upregulated and 44 miRNAs downregulated in brain I/R injury. The target genes of these differentially expressed miRNAs are mainly involved in metabolic and cellular processes and are recognized as pivotal nodes of miRNA-gene ontology (GO) networks. Another study also found that miR-21 and miR-29b both have upregulation effects in hypoxic and glucose-deficient neurons and astrocytes, and these neurons and astrocytes simulate $\mathrm{I} / \mathrm{R}$ in vitro [31]. Both studies emphasized the role of miRNAs in the pathogenesis of brain $\mathrm{I} / \mathrm{R}$ injury. However, the exact role and regulatory mechanism of miRNAs in brain $\mathrm{I} / \mathrm{R}$ injury remain unclear.

A recent study reported that miR-146a is upregulated in the brain tissue of mice with experimental cerebral $I / R$ injury. Inhibition of miR-146a results in upregulation of expression of interleukin receptor-related kinase-1 (IRAK1). IRAK1 aggravates neurobehavioral disorders caused by I/R, which expands the infarct scope, aggravates blood-brain barrier leakage, and intensifies brain I/R-induced neuronal cell death by activating the nuclear factor kappa $\mathrm{B}(\mathrm{NF}-\kappa \mathrm{B})$ signaling pathway and nuclear accumulation of p65. Therefore, we hypothesize that miR-146a may be a potential target for the brain $\mathrm{I} / \mathrm{R}$ injury treatment [32].

3.3. Hepatic Ischemia/Reperfusion Injury. Hepatic I/R injury is a common complication of liver transplantation and general anesthesia [33]. miR-370 is a marker with important functions in several human tumors, including gastric cancer [34], liver cancer [35], prostate cancer [36], and laryngeal squamous cell carcinoma [37]. In recent years, researchers have found that miR-370 is also a promising noninvasive biomarker for patients with liver I/R injury. Li et al. [38] found that miR-370 levels increased after liver I/R. Inhibition of miR-370 can reduce the expression of serum transaminase and proinflammatory cytokines and improve histological damage in the liver. They also confirmed that this mechanism may be linked to miR-370 targeting of the $3^{\prime}$-UTR of transforming growth factor- $\beta$ receptor II (TGF $\beta$ RII) with a potential role in hepatic I/R injury. Meanwhile, Zare et al. [39] found that bone marrow-derived mesenchymal stem cell (BM-MSCs) had a stronger effect on liver I/R mice than ischemic preconditioning. In addition, in BM-MSCs, downregulating the inhibitory effect of miR370 on liver injury may play a role by decreasing the expres- sion of Bax and upregulating Bcl2. However, the exact regulatory mechanism of miR-370 in hepatic I/R injury is still not fully understood, and further research is needed to clarify the mechanism. Interestingly, the expression level of miR-370 also has a protective effect against MI/RI [40]. Therefore, it can be concluded that the effect of miR-370 is stimulus- and cell/tissue-type dependent.

3.4. Renal Ischemia/Reperfusion Injury. Renal I/R injury may be related to surgical treatment and is a common phenomenon in kidney transplantation [41, 42]. On one hand, renal injury caused by $I / R$, especially acute renal failure, is closely related to hypoxia and inflammatory reaction [43] which promotes endothelial cell activation, injury, and leukocyte adhesion, triggers leukocyte clamping, and damages microvascular blood flow. On the other hand, the initial nonimmune hypoxia injury and subsequent reperfusion will also activate the natural immune response, resulting in different degrees of tissue damage. The prognosis of AKI is poor because there is no effective treatment or prevention of I/ $\mathrm{R}$-induced AKI. Therefore, there is an urgent need to develop an effective method to treat AKI caused by I/R. Study has shown that preventing the execution of mitochondrial fission and maintaining mitochondrial homeostasis is a potential treatment [44]. miRNAs are important regulators of gene expression. They can silence genetic information after transcription by targeting the $3^{\prime}$-UTR of mRNAs and eventually inhibit protein synthesis or increase mRNA degradation.

Wang et al. [45] detected miRNAs in the kidney $12 \mathrm{~h}$ after renal I/R injury with an miRNA chip and determined that 36 miRNAs were abnormally expressed in the kidneys of rats with renal I/R injury, of which 15 miRNAs showed significant change. Quantitative real-time PCR results were also consistent with the chip results. Notably, miR-10a, miR-192, and miR-194 in the plasma of rats with renal I/R injury were significantly increased, of which miR-10a was increased within $1 \mathrm{~h}$ of reperfusion, and the peak time of miR-192 and miR-194 is similar to that of serum creatinine $6 \mathrm{~h}$ after reperfusion. Therefore, miR-10a in plasma may be the best biomarker for renal I/R injury in rats.

Zou et al. [46] quantitative determination of miR-30c$5 p$, miR-378a-3p, and miR-192-5p levels in the urine of rats and AKI patients confirmed miR-30c-5p and miR-192-5p as possible diagnostic markers of I/R-induced AKI. Previous studies have shown that hypoxia inducible factor- $1 \alpha$ $(\mathrm{HIF} 1 \alpha)$ is a key transcription factor that protects cells from hypoxic injury $[47,48]$. miR-30c-5p is upregulated in an $\mathrm{H} /$ $\mathrm{R}$ model and can protect epithelial cells from anoxic damage, whereas HIF $1 \alpha$ obviously promotes this protective effect. In addition, miR-30c-5p stabilizes the expression level of HIF $1 \alpha$ by targeting suppressor of cytokine signaling- 3 (SOCS3) [46]. Some studies have confirmed that renal I/R injury can activate critical inflammatory cascades, including activation of NF- $\kappa B[49,50]$. In the process of renal I/R injury, oxidative stress and inflammatory reactions are manifested by activation of the HIF $1 \alpha$ hypoxia and NF- $\kappa \mathrm{B}$ inflammation signaling pathways, whereas transcription of most inflammatory factors and adhesion factors are 
regulated by both of these. A study showed that HIF $1 \alpha$ and $\mathrm{NF}-\kappa \mathrm{B}$ have synergistic effects in the treatment of AKI [51]. Since the NF- $\kappa \mathrm{B}$ signaling pathway is an important target for miRNAs to respond to renal $I / R$, elucidating whether NF- $\kappa \mathrm{B}$ activity is regulated by miR-30c-5p could be of interest.

3.5. Intestine Ischemia/Reperfusion Injury. Intestinal I/R injury is an important problem in abdominal aortic aneurysm surgery, small bowel transplantation, extracorporeal circulation, strangulated hernia, and neonatal necrotizing enterocolitis [52]. Among the pattern recognition receptors, toll-like receptor (TLR) is the main sensor that produces an inflammatory network and is also a key regulator of the natural immune system [53]. Uncontrolled activation of the natural immune system by TLR plays a key role in I/R-mediated tissue injury. Tumor necrosis factor- $\alpha$ (TNF $\alpha)$ and interleukin-1 $\beta$ (IL-1 $\beta$ ) are the two most effective cytokines secreted during TLR ligand stimulation of the natural immune activation process [54]. IL- $1 \beta$ signaling induces the NF- $\kappa \mathrm{B}$ signaling pathway through activation of myeloid differentiation primary response-88 (MyD88), IL-1R-related kinases (IRAK1, IRAK2, IRAK4), TNF-related associated factor-6 (TRAF6), and TGF $\beta$-activated kinases, thus immediately and temporarily activating normal physiological responses to pathogens.

miR-146a is considered to have a negative effect on the innate immune response $[55,56]$. Chassin et al. [57] used mouse intestinal I/R injury models and human intestinal mucosa biopsy to observe the enhanced expression of the TLR signaling molecule IRAK1 protein in ischemic epithelial cells and found that its reactivity to natural immune stimulation was significantly enhanced. When miR-146a is induced by $3,3^{\prime}$-diinodolylmethane (DIM), it controls the level of epithelial IRAK1 protein in mouse and human mucosal tissues through translation inhibition and protection of I/R injury. This indicates the application or pharmacological induction of miR-146a as a new strategy to control natural immune hyperreactivity and reduce transient hypoxia or tissue injury after I/R.

miR-146a improves the survival of intestinal epithelial cells under ischemia and I/R injury by inhibiting TLR4, TRAF6, and phosphorylation of NF- $\kappa$ light polypeptide gene enhancer in B-cells inhibitor- $\alpha(\mathrm{p}-\mathrm{I} \kappa \mathrm{B} \alpha)$, thus leading to a decrease in nuclear translocation of NF- $\kappa \mathrm{B}$ p 65 , an increase in cell survival rate, and a decrease in cell apoptosis [58].

\section{Diagnostic Biomarkers of $I / R$}

In the past, endothelial cell injury and troponin have been used as markers of $\mathrm{I} / \mathrm{R}$ injury $[59,60]$, but the progression and prognosis of the disease cannot be accurately determined by the degree of endothelial cell injury, while the delayed release time of troponin often leads to low sensitivity. Recently, differential changes in miRNAs in I/R injury have been confirmed. Given the specificity, timing, and relative conservativeness of miRNA expression, miRNAs have great potential as biomarkers. miRNAs showed high conservation in sequences of different species, which promotes the translation from animal models to humans. miRNAs have high tissue specificity, which is an obvious imbalance in many human diseases [61]. In addition, they are stable in tissues and body fluids (such as plasma, serum, and exosomes), which make miRNAs suitable as repositories for biomarker discovery.

Rensen et al. [62] performed miRNA sequence analysis using microarray analysis and quantitative polymerase chain reaction (qPCR) techniques in groups of patients with either acute ischemic stroke (AIS) (10 cases) or neurological diseases (10 cases). A total of 183 different miRNAs were detected in the cerebrospinal fluid, of which two miRNAs (let-7c and miR-221-3p) were both associated with stroke. A total of 287 different miRNAs were detected in the blood, of which two miRNAs (miR-151 a-3p and miR-140-5p) were upregulated and one miRNA (miR-18b-5p) was downregulated in the stroke group.

Xue et al. [63] proved for the first time that miR-26a-1, miR-146, and miR-199a-1 can be used as candidate biomarkers for AMI. The expression levels of miR-26a-1, miR-146, and miR-199a-1 in AMI patients were significantly higher than those in normal individuals. Meanwhile, the expression levels of three kinds of miRNAs in AMI patients before and after percutaneous coronary intervention (PCI) were significantly higher than those in the control group. The combined detection of three kinds of miRNAs had higher AUC values before and after PCI, suggesting that the combined application of miRNAs has higher accuracy in the diagnosis of AMI.

No miR-519e-5p was detected in the plasma of healthy volunteers and non-AMI patients (IS patients and pulmonary embolism (PE) patients), but the levels of three circulating miRNAs in IS and PE were all increased, while the plasma miR-519e-5p level was only detected in AMI [64]. Circulating miR-21-5p and miR-361-5p (especially miR361-5p) showed an upward trend similar to that of plasma cardiac troponin I (cTnI) in early AMI (0-4h). Circulating miR-519e-5p levels are relatively low in AMI patients, which is contrary to the expression in other ischemic diseases. These results indicate circulating miR-519e-5p as an effective biomarker for distinguishing acute AMI from PE and IS. Recently, a study showed that miR-423-5p can be used as a useful biomarker for clinical ischemic diseases. Rizzacasa et al. [65] reported that miR-423-5p may be related to the risk and severity of either stable or unstable coronary heart disease (CHD) 6 months after AMI. miR-423-5p provides a new genetic marker for the early prevention and intervention of CHD.

Schulte et al. [66] proved that miR-126, miR-197, and miR-223 derived from serum participate in intravascular inflammation and platelet activation and may be considered as biomarkers of cardiovascular death in patients with CHD. Jickling et al. [67] compared the levels of miRNAs between the peripheral blood of 24 patients with IS and 24 patients with risk factors. Microarray analysis showed that the expression levels of six miRNAs were downregulated (miR122, miR-148A, let-7i, miR-19a, miR-320d, miR-4429), and two were upregulated (miR-363 and miR-487b).

Zhou et al. [68] reported that high levels of miR-134 can be detected in serum samples of patients with AIS within 
$24 \mathrm{~h}$, which may be suitable as a potential genetic marker of the disease. Zhang et al. [69] found that the plasma miR-192 levels in patients with AKI were significantly higher than those in patients without AKI $2 \mathrm{~h}$ after admission. It is suggested that the dynamic change of miR-192 in plasma levels in patients with AKI may be a predictor of early ischemic AKI.

In summary, miRNAs associated with $\mathrm{I} / \mathrm{R}$ injury are promising biomarkers for identifying ischemic diseases and assessing their prognosis (Table 1). With the development of qPCR, miRNAs have been widely used in the translation field [70]. The I/R-related diseases discussed above were not diagnosed at the onset of a large number of studies; the diagnostic value of these miRNAs remains to be determined in future. Therefore, to apply these miRNAs in clinical practice, clinical trials with large sample sizes and longterm follow-up are needed.

\section{Targeted miRNAs Treating I/R Injury}

Studies have found that correcting the expression of one or several key genes in I/R injury can restore the physiological expression levels of damaged genes, which provides an opportunity for the molecular-targeted therapy of ischemic diseases. As a key regulator of gene expression, miRNAs not only open up a new research field for biomarker research of ischemic diseases but also for the treatment of ischemic diseases [71]. To date, several methods have been developed to down- or upregulate miRNAs, among which miRNA mimetics and inhibitors have shown promise as new therapeutic drugs in clinical application [72].

5.1. Anti-miRNA Oligodeoxynucleotide Technology. The most effective inhibitor of miRNA is anti-miRNA oligonucleotides (AMO), which can combine with miRNA, inhibit the combination of endogenous miRNA and mRNA, and relieve the inhibitory effect of miRNA on mRNA. AMO is unstable and easily degraded in vivo; therefore, various chemical modifications are used to improve the stability of AMO in vivo. These include locked nucleic acid (LNA), 2' -fluoro-RNA (2'-F-RNA), 2' -O-methoxyethyl (MOE), peptide nucleic acid (PNA), and phosphorodiamidate morpholino oligonucleotides (PMOs). Properly designing a specific AMO can improve the performance and efficiency of targeting miRNAs by increasing nuclease resistance and target affinity [73].

AMO can be a potential therapeutic method by inhibiting disease-related miRNAs. In the treatment of I/R-related diseases, ischemic postconditioning (IPost) can downregulate relevant miRNAs to protect the heart from infarction after reperfusion. Tu et al. [74] introduced 2-O methyl AMO complementary to miR-21 sequence into mouse myocardium and found that using Antagomir-21 instead of an interfering agent can reduce the protective effect of post on mouse MI/RI. In addition, after reperfusion for $3 \mathrm{~h}$, Antagomir-21 drug-treated hearts showed a significant decrease in cardiac function recovery, showing a decrease in left ventricular ejection fraction (EF) and fractional shortening (FS) and an increase in left ventricular end diastolic diameter (LVEDD) and left ventricular end-systolic diameter (LVESD).

Shen et al. [75] found that the infusion of LNA-miR-30 family inhibitor can correspondingly increase the levels of cystathionine-gamma-lyase (CSE) and hydrogen sulfide $\left(\mathrm{H}_{2} \mathrm{~S}\right)$ after AMI, reduce the infarct area, reduce the number of apoptotic cells in the infarct area, and improve cardiac function after AMI. There are also reports of in vivo studies, using intravenous injection or tissue local injection, that evaluated the feasibility and effectiveness of miRNA application as a treatment.

5.2. siRNA Gene Knockout Technology. Small interfering RNA (siRNA) is a chemically synthesized dsRNA with a length of 19-23 nt. To function, an siRNA must be absorbed by specific cells and enter the cytoplasm. siRNAs are contained in RISC and transferred to the Argonaute protein of the RISC catalytic core. Protein-RNA complexes separate dsRNA and discard the passenger strands [76, 77]. Then, RISC, guided by the antisense strand, downregulates the expression of precise genes by either degrading specific mRNA or physically blocking mRNA translation $[78,79]$.

siRNA-mediated silencing has been proven to be effective in targeting miRNAs of interest in mice. For example, siRNA knockout of lethal-7 (let-7) can effectively inhibit the expression of let-7 in nerve cells, thereby reducing apoptosis and neurological deficits after cerebral I/R injury [80]. A recent study showed that mice treated with necrosis-related factor (Nrf) siRNA showed a decrease in the AMI area and an improvement in cardiac function during MI/RI [81]. These studies show that downregulation of miRNAs through siRNAs may be a promising tool for the treatment of ischemic diseases.

5.3. miRNA Sponge Technology. miRNA sponges are RNA molecules with a plurality of miRNAs in series, which can separate the required miRNAs from the target miRNAs [82]. Endogenous miRNA sponges, also known as competing endogenous RNAs (ceRNAs), act to buffer the activity of miRNAs on physiologically related targets. Its principle is dominant negative regulation, and it can efficiently inhibit miRNA action for a prolonged time [83]. The application of miRNA sponges in the treatment of ischemic diseases has only just begun. Li et al. [84] infected miRNA sponges with miR-497 binding site and adenovirus with recombinant plasmid into neonatal rat cardiomyocytes (NRCS) under aortic regurgitation (AR) and left ventricular myocardium of mice under I/R. Studies have found that miRNA sponges inhibiting miR-497 can upregulate the expression of the antiapoptosis gene $B c l 2$ and autophagy gene $L C 3 B$, significantly reduce cell apoptosis, and enhance autophagic flux. In addition, the effectiveness of miRNA sponges can be evaluated by detecting the expression of the target genes with a fluorescence reporting system. Generally, if there is a target miRNA, the fluorescence reporting system should be significantly inhibited by miRNA sponges.

At present, miRNA sponges are mainly used in the experimental field of miRNA function research, while their clinical application still needs further evaluation. miRNA 
TABLE 1: miRNAs associated with I/R injury as a clinical biomarker of ischemic diseases.

\begin{tabular}{lccccc}
\hline miRNA & Disease & Cases & Controls & Source & Reference \\
\hline let-7c, miR-221-3p, miR-151a-3p, miR-140-5p, miR-18b-5p & AIS & 20 & 10 & Cerebrospinal fluid blood & {$[62]$} \\
miR-26a-1, miR-146a, miR-199a-1 & AMI & 58 & 27 & Plasma & {$[63]$} \\
miR-519e-5p & AMI & 45 & 28 & Plasma & {$[64]$} \\
miR-423-5p & CHD, AMI & 99 & 38 & Plasma, PBMCs & {$[65]$} \\
miR-126, miR-197, miR-223 & CHD & 873 & $340 / 533$ & Serum & {$[66]$} \\
miR-134 & AIS & 100 & 50 & Serum & {$[68]$} \\
\hline
\end{tabular}

sponge technology has many advantages, including equivalent efficacy to AMO, and is also simpler than gene knockout with application in various cell lines and models. Furthermore, miRNA sponges only affect mature mRNAs and can inhibit the action of a specific miRNA without affecting other miRNAs. However, in the field of ischemic disease treatment, antisense technology may be more promising pending the continuous development and improvement of antisense technology and transfer of oligonucleotides into cells.

5.4. Peptide Nucleic Acid Technology. PNA is a synthetic ana$\log$ of DNA, which was introduced by Nielsen et al. [85] in 1991. Anti-miRNA PNA can bind miRNAs through steric hindrance and inhibit their interaction with RISC, thereby reducing the expression of miRNAs [86]. PNA has a number of advantages over other anti-miRNA substances, such as not having to target full-length miRNAs. Thus, PNA is a good candidate for biomedical applications [87]. Studies have shown that PNAs have the ability to efficiently inhibit miR-155, which is expressed in the hematopoietic system in primary B cells as well as in a mouse model of lymphoma $[88,89]$. A study has shown that intraocular injection of antisense vascular endothelial growth factor (Vegf) PNA or antisense Ephrin type-A receptor 2 (EphA2) PNA can significantly reduce their respective target mRNAs in ischemic retinas and inhibit retinal neovascularization [90]. Downregulation of miRNA by PNA is expected to be an effective intervention for ischemic diseases. However, more data are needed on its potential side effects or toxicity.

5.5. miRNA Overexpression Vectors. In recent years, viral transfection (including lentivirus and adenovirus) has continued to occupy a central position in gene therapy as transgenic vectors, with high transfection efficiency. Using different viral vector tools can realize efficient and stable transduction of cells and animals and gradually become a substitute for miRNA overexpression plasmids. For example, $\mathrm{Qu}$ et al. [91] found that the increased overexpression of miRNA-126 promoted cerebrovascular generation and neurogenesis, which further improved neurobehavioral results in ischemic mice. Wang et al. [92] administered lentivirus carrying miR-214 to reduce the area of AMI after I/R injury by targeting the expression of Bril-Ems-Suppressor1(BES1-) interacting Myc-like protein-1 (Bim1) and preventing its transfer from cytoplasm to mitochondria in vivo and vitro. This significantly reduced the apoptosis of myocardial cells induced by $\mathrm{I} / \mathrm{R}$ and further protected the heart from I/R injury without toxicity.

Overexpression of traditional viral vectors is usually systemic rather than specific to target cells or tissues. However, various adenoviral vector systems have shown improve targeting for gene delivery, which are expected to be used in gene therapy and treatment of diseases related to I/R. These vectors are easy to handle, have a wide range of tropism, and have a high titer capability. Furthermore, they have been optimized to avoid unnecessary side effects and target specific tissues or cell types [93].

5.6. miRNA Mimetics. miRNA mimetics are small, chemically modified dsRNAs that mimic mature miRNAs in vivo, which have been widely used in functional experiments. Selvamani and Sohrabji [94] successfully inhibited the expression of caspase- 3 in neuronal cells of female stroke patients through synthetic miR-363 mimetics targeting overexpressed caspase- 3 protein. Although miR-363 therapy has a strong neuroprotective effect on middle-aged women, it does not reduce the infarct volume of men or improve sensorimotor function. The mi363-3p mimetic is located in neurons and may reduce the infarct volumen by influencing on the apoptosis process. miR-144 has strong acute myocardial protection in I/R injury models. Zhou et al. [95] proved that intravenous injection of miR-144 mimetics has a strong effect on remodeling after AMI, which indicate that miR-144 has a potential therapeutic effect after AMI.

Many miRNA mimetics, such as pre-miRNA precursor (Ambion) and miRIDIANTM microRNA mimetic (Thermo Scientific Dharmacon), have been commercialized. Because miRNA mimetics have no carrier toxicity, if the drug delivery system was verified to have no side effects after long-term use, it will be a promising therapy for anti-ischemic diseases.

\section{Discussion}

As a relatively new research field in ischemic diseases, miRNAs have attracted attention with broad application prospects. The therapies based on miRNA have achieved remarkable results in various $I / R$ injury in vivo and in vitro. The role of miRNAs in diagnosis, changes, and prognosis of different types of ischemic diseases is exciting. It is noteworthy that the expression level of miRNAs in plasma, serum, and blood cells in patients with ischemic diseases has changed greatly, which emphasizes the potential role of miRNAs in the diagnosis and treatment of ischemic 
diseases. With further in-depth study about the mechanism of miRNAs in the occurrence and development of diseases, some treatment methods based on miRNA will change human disease treatment dramatically. Once the problems and challenges of miRNAs in the ischemic disease treatments are solved, miRNAs will become one of prominent candidates competing with protein inhibitors. However, some technical and practical difficulties still need to be solved before they can be converted into clinical practice.

Increasing evidence shows that miRNAs are extensively involved in I/R injury. For instance, miRNAs play a crucial role in $\mathrm{I} / \mathrm{R}$ injury and regulation of the inflammatory NF$\kappa \mathrm{B}$ signaling pathway. The lack of conservativeness of miRNA sequences between different species in basic research studies is still an obstacle to its clinical application. These miRNA-related therapies are effective in humans, and its potential adverse reactions remain to be answered. In addition, the regulation of miRNAs on target genes affects their transcription or degradation, as well as the regulation of other expressed miRNAs after returning to the nucleus, and the functions of exogenous miRNAs in cell-to-cell communication may be different between animals and humans. Therefore, the in-depth preclinical research should be performed on targeted diseases to determine their functional characteristics before patients receive treatment.

In summary, this study highlighted the broad applications of miRNAs I/R injury and elaborated on targeted therapies using gene expression regulation methods. These included antisense oligonucleotides and siRNA gene knockout techniques, miRNA sponges, PNA techniques, miRNA overexpression vectors, and miRNA mimics which interfere with miRNA expression and their application to treat I/R injury. Currently, many of these technologies have shortcomings with stability and delivery as the most common problems, which are potential reasons why miRNAs have not yet reached clinical translation. Only when miRNAs are reasonably designed and combined with existing clinical treatment methods can more effective disease treatment schemes be formed. This review provided new research results obtained in this research field. It is hoped that new research on this topic will not only generate new insights into the pathophysiology of miRNA in I/R injury but also can provide a basis for the clinical application of miRNA in I/R. There is no doubt that pursuing this course of treatment will be long, making it challenging. Therefore, it is prudent to continue to explore miRNAs as potential therapies for I/R injury.

\section{Abbreviations}

$\begin{array}{ll}2^{\prime} \text {-F-RNA: } & 2^{\prime} \text {-Fluoro-RNA } \\ \text { Ago2: } & \text { Argonaute-2 } \\ \text { AIS: } & \text { Acute ischemic stroke } \\ \text { AKI: } & \text { Acute kidney injury } \\ \text { AMI: } & \text { Acute myocardial infarction } \\ \text { AMO: } & \text { Anti-miRNA oligonucleotides } \\ \text { AR: } & \text { Aortic regurgitation } \\ \text { Bax: } & \text { Bcl2-associated X protein } \\ \text { Bcl2: } & \text { B-cell lymphoma-2 } \\ \text { BES1: } & \text { Bri1-Ems-Suppressor1 } \\ \text { Bim1: } & \text { BES1-interacting Myc-like protein-1 }\end{array}$

BM-MSC: Bone marrow-derived mesenchymal stem cell

CHD: $\quad$ Coronary heart disease

CBA: $\quad$ Chicken $\beta$-actin

ceRNA: Competitive endogenous RNA

CSE: Cystathionine-gamma-lyase

cTnI: $\quad$ Cardiac troponin I

DIM: $\quad 3,3^{\prime}$-Diinodolylmethane

dsRNA: Double-stranded RNA

EphA2: $\quad$ Ephrin type-A receptor 2

EF: $\quad$ Ejection fraction

Fas: $\quad$ Fatty acid synthase

FS: $\quad$ Fractional shortening

H/R: Hypoxia/reoxygenation

H2S: Hydrogen sulfide

HIF1 $\alpha$ : $\quad$ Hypoxia inducible factor- $1 \alpha$

IL-1 $\beta$ : Interleukin-1 $\beta$

IPO8: $\quad$ Importin-8

IPost: Ischemic postconditioning

IRAK: Interleukin-1 receptor-associated kinase

IS: $\quad$ Ischemic stroke

LC3-II: $\quad$ Light chain 3-II

let-7: $\quad$ Lethal-7

LNA: $\quad$ Locked nucleic acid

LVEDD: Left ventricular end diastolic diameter

LVESD: Left ventricular end-systolic diameter

MI/RI: Myocardial ischemia reperfusion injury

miRNA: MicroRNA

miRNA $*$ : MicroRNA passenger chain

MOE: $\quad 2$ '-O-methoxyethyl

MyD88: $\quad$ Myeloid differentiation primary response-88

NF- $\kappa$ B: $\quad$ Nuclear factor kappa B

NRCS: Neonatal rat cardiomyocytes

Nrf: Necrosis-related factor

PE: $\quad$ Pulmonary embolism

$\mathrm{p}-\mathrm{I} \kappa \mathrm{B} \alpha$ : $\quad$ Phosphorylation of NF- $\kappa$ light polypeptide

gene enhancer in B-cells inhibitor- $\alpha$

PCI: Percutaneous coronary intervention

PMO: $\quad$ Phosphorodiamidate morpholino

oligonucleotide

PNA: $\quad$ Peptide nucleic acid

pre-miRNA: miRNA precursor

pri-miRNA: Primary miRNA

qPCR: Quantitative polymerase chain reaction

RISC: $\quad$ RNA-induced silencing complex

ROS: $\quad$ Reactive oxygen species

siRNA: Small interfering RNA

SOCS3: $\quad$ Suppressor of cytokine signaling-3

TGF $\beta$ RII: $\quad$ Transforming growth factor- $\beta$ receptor II

TLR: Toll-like receptor

TNF $\alpha$ : Tumor necrosis factor- $\alpha$

TRAF6: $\quad$ TNF receptor associated factor- 6

UTR: Untranslated region

Vegf: $\quad$ Vascular endothelial growth factor.

\section{Conflicts of Interest}

The authors declare that there is no conflict of interest regarding the publication of this paper. 


\section{Authors' Contributions}

MingMing Cao and Luwen Zhu designed the study. Mingming Cao and Wenjing Song wrote the manuscript. Runyu Liang and Luwen Zhu revised the manuscript. Lili Teng, Mei Zhang, and Jiyao Zhang participated in material collection. All authors read and approved the final manuscript. MingMing Cao and Wenjing Song contributed equally to this work.

\section{Acknowledgments}

This work was supported by the Natural Science Foundation of Heilongjiang (LH2020H083), the University Nursing Program for Young Scholar with Creative Talents in Heilongjiang Province (UPYSCT-2018226), and the Research Fund Project of Heilongjiang University of Chinese Medicine (2015bs02).

\section{References}

[1] X. L. Min, T. Y. Wang, Y. Cao, J. Liu, J. T. Li, and T. H. Wang, "MicroRNAs: a novel promising therapeutic target for cerebral ischemia/reperfusion injury?," Neural Regeneration Research, vol. 10, no. 11, pp. 1799-1808, 2015.

[2] M. Bhaskaran and M. Mohan, "MicroRNAs: history, biogenesis, and their evolving role in animal development and disease," Veterinary Pathology, vol. 51, no. 4, pp. 759-774, 2014.

[3] J. B. Weiss, S. U. Eisenhardt, G. B. Stark, C. Bode, M. Moser, and S. Grundmann, "MicroRNAs in ischemia-reperfusion injury," American Journal of Cardiovascular Disease, vol. 2, no. 3, pp. 237-247, 2012.

[4] F. Afonso-Grunz and S. Müller, "Principles of miRNA-mRNA interactions: beyond sequence complementarity," Cellular and Molecular Life Sciences, vol. 72, no. 16, pp. 3127-3141, 2015.

[5] L. F. Gulyaeva and N. E. Kushlinskiy, "Regulatory mechanisms of microRNA expression," Journal of Translational Medicine, vol. 14, no. 1, p. 143, 2016.

[6] M. T. Bohnsack, K. Czaplinski, and D. Gorlich, "Exportin 5 is a RanGTP-dependent dsRNA-binding protein that mediates nuclear export of pre-miRNAs," RNA, vol. 10, no. 2, pp. 185-191, 2004.

[7] S. M. Mah, C. Buske, R. K. Humphries, and F. Kuchenbauer, "miRNA: a passenger stranded in RNA-induced silencing complex?," Critical Reviews in Eukaryotic Gene Expression, vol. 20, no. 2, pp. 141-148, 2010.

[8] S. L. Ameres and P. D. Zamore, "Diversifying microRNA sequence and function," Nature Reviews. Molecular Cell Biology, vol. 14, no. 8, pp. 475-488, 2013.

[9] L. Zeng, Y. Chen, Y. Wang et al., "MicroRNA hsa-miR-370-3p suppresses the expression and induction of CYP2D6 by facilitating mRNA degradation," Biochemical Pharmacology, vol. 140, pp. 139-149, 2017.

[10] H. W. Liang, J. Zhang, K. Zen, C. Y. Zhang, and X. Chen, "Nuclear microRNAs and their unconventional role in regulating non-coding RNAs," Protein \& Cell, vol. 4, no. 5, pp. 325-330, 2013.

[11] Y. Wei, L. Li, D. Wang, C. Y. Zhang, and K. Zen, "Importin 8 Regulates the Transport of Mature MicroRNAs into the Cell Nucleus," Journal of Biological Chemistry, vol. 289, no. 15, pp. 10270-10275, 2014.
[12] A. Montecalvo, A. T. Larregina, W. J. Shufesky et al., "Mechanism of transfer of functional microRNAs between mouse dendritic cells via exosomes," Blood, vol. 119, no. 3, pp. 756766, 2012.

[13] A. Muthusamy, C. M. Lin, S. Shanmugam, H. M. Lindner, S. F. Abcouwer, and D. A. Antonetti, "Ischemia-reperfusion injury induces occludin phosphorylation/ubiquitination and retinal vascular permeability in a VEGFR-2-dependent manner," Journal of Cerebral Blood Flow and Metabolism, vol. 34, no. 3, pp. 522-531, 2014.

[14] J. Nanobashvili, C. Neumayer, A. Fuegl et al., "Development of 'no-reflow' phenomenon in ischemia/reperfusion injury: failure of active vasomotility and not simply passive vasoconstriction," European Surgical Research, vol. 35, no. 5, pp. 417-424, 2003.

[15] K. Szocs, "Endothelial dysfunction and reactive oxygen species production in ischemia/reperfusion and nitrate tolerance," General Physiology and Biophysics, vol. 23, no. 3, pp. 265295, 2004.

[16] H. Dehaini, H. Awada, A. el-Yazbi et al., "MicroRNAs as potential pharmaco-targets in ischemia-reperfusion injury compounded by diabetes," Cells, vol. 8, no. 2, p. 152, 2019.

[17] T. Inoue, "Ischemia-reperfusion injury is still a big hurdle to overcome for treatment of acute myocardial infarction," Journal of Cardiology, vol. 67, no. 4, pp. 305-306, 2016.

[18] H. Zhao, R. M. Sapolsky, and G. K. Steinberg, "Interrupting reperfusion as a stroke therapy: ischemic postconditioning reduces infarct size after focal ischemia in rats," Journal of Cerebral Blood Flow and Metabolism, vol. 26, no. 9, pp. 1114-1121, 2006.

[19] E. Hitchman, R. B. Hitchman, and L. A. King, "BacMam delivery of a protective gene to reduce renal ischemia-reperfusion injury," Human Gene Therapy, vol. 28, no. 9, pp. 747-756, 2017.

[20] S. Ryan, C. T. Taylor, and W. T. McNicholas, "Selective activation of inflammatory pathways by intermittent hypoxia in obstructive sleep apnea syndrome," Circulation, vol. 112, no. 17, pp. 2660-2667, 2005.

[21] N. L. Halladin, S. Ekeløf, M. Alamili et al., "Lower limb ischaemia and reperfusion injury in healthy volunteers measured by oxidative and inflammatory biomarkers," Perfusion, vol. 30, no. 1, pp. 64-70, 2015.

[22] K. L. Wallace and J. Linden, "Adenosine A2A receptors induced on iNKT and NK cells reduce pulmonary inflammation and injury in mice with sickle cell disease," Blood, vol. 116, no. 23, pp. 5010-5020, 2010.

[23] I. Bougioukas, V. Didilis, J. Emigholz et al., "The effect of amifostine on lung ischaemia-reperfusion injury in rats," Interactive Cardiovascular and Thoracic Surgery, vol. 23, no. 2, pp. 273-279, 2016.

[24] M. Neri, I. Riezzo, N. Pascale, C. Pomara, and E. Turillazzi, "Ischemia/reperfusion injury following acute myocardial infarction: a critical issue for clinicians and forensic pathologists," Mediators of Inflammation, vol. 2017, Article ID 7018393, 14 pages, 2017.

[25] S. Dong, Y. Cheng, J. Yang et al., "MicroRNA Expression Signature and the Role of MicroRNA-21 in the Early Phase of Acute Myocardial Infarction," Journal of Biological Chemistry, vol. 284, no. 43, pp. 29514-29525, 2009.

[26] J. Xiao, Y. Pan, X. H. Li et al., "Cardiac progenitor cell-derived exosomes prevent cardiomyocytes apoptosis through 
exosomal miR-21 by targeting PDCD4," Cell Death \& Disease, vol. 7, no. 6, article e2277, 2016.

[27] I. Jeremias, C. Kupatt, A. Martin-Villalba et al., "Involvement of CD95/Apo1/Fas in cell death after myocardial ischemia," Circulation, vol. 102, no. 8, pp. 915-920, 2000.

[28] P. Lee, M. Sata, D. J. Lefer, S. M. Factor, K. Walsh, and R. N. Kitsis, "Fas pathway is a critical mediator of cardiac myocyte death and MI during ischemia-reperfusion in vivo," American Journal of Physiology Heart and Circulatory Physiology, vol. 284, no. 2, pp. H456-H463, 2003.

[29] S. F. He, H. J. Zhu, Z. Y. Han et al., "MicroRNA-133b-5p Is Involved in Cardioprotection of Morphine Preconditioning in Rat Cardiomyocytes by Targeting Fas," Canadian Journal of Cardiology, vol. 32, no. 8, pp. 996-1007, 2016.

[30] R. V. Krishnamurthi, A. E. Moran, V. L. Feigin et al., "Stroke prevalence, mortality and disability-adjusted life years in adults aged 20-64 years in 1990-2013: data from the Global Burden of Disease 2013 Study," Neuroepidemiology, vol. 45, no. 3, pp. 190-202, 2015.

[31] M. T. Ziu, L. Fletcher, S. Rana, D. F. Jimenez, and M. Digicaylioglu, "Temporal differences in microRNA expression patterns in astrocytes and neurons after ischemic injury," PLoS One, vol. 6, no. 2, article e14724, 2011.

[32] B. Chu, Y. Zhou, H. Zhai, L. Li, L. Sun, and Y. Li, "The role of microRNA-146a in regulating the expression of IRAK1 in cerebral ischemia-reperfusion injury," Canadian Journal of Physiology and Pharmacology, vol. 96, no. 6, pp. 611-617, 2018.

[33] Y. Li, Y. Yang, Y. Feng et al., "A review of melatonin in hepatic ischemia/reperfusion injury and clinical liver disease," Annals of Medicine, vol. 46, no. 7, pp. 503-511, 2014.

[34] C. Fan, S. Liu, Y. Zhao et al., "Upregulation of miR-370 contributes to the progression of gastric carcinoma via suppression of FOXO1," Biomedicine \& Pharmacotherapy, vol. 67, no. 6, pp. 521-526, 2013.

[35] G. Sun, Y. B. Hou, H. Y. Jia, X. H. Bi, L. Yu, and D. J. Chen, "MiR-370 promotes cell death of liver cancer cells by Akt/ FoxO3a signalling pathway," European Review for Medical and Pharmacological Sciences, vol. 20, no. 10, pp. 2011-2019, 2016.

[36] Z. Wu, H. Sun, W. Zeng, J. He, and X. Mao, "Upregulation of MircoRNA-370 induces proliferation in human prostate cancer cells by downregulating the transcription factor FOXO1," PLoS One, vol. 7, no. 9, article e45825, 2012.

[37] W. Yungang, L. Xiaoyu, T. Pang, L. Wenming, and X. Pan, "miR-370 targeted FoxM1 functions as a tumor suppressor in laryngeal squamous cell carcinoma (LSCC)," Biomedicine \& Pharmacotherapy, vol. 68, no. 2, pp. 149-154, 2014.

[38] L. Li, G. Li, C. Yu et al., "A role of microRNA-370 in hepatic ischaemia-reperfusion injury by targeting transforming growth factor- $\beta$ receptor II," Liver International, vol. 35, no. 4, pp. 1124-1132, 2015.

[39] M. A. Zare, A. Zare, N. Azarpira, and S. Pakbaz, "The protective effect of bone marrow-derived mesenchymal stem cells in liver ischemia/reperfusion injury via down-regulation of miR370," Iranian Journal of Basic Medical Sciences, vol. 22, no. 6, pp. 683-689, 2019.

[40] H. Liu, N. Yang, Z. Fei et al., "Analysis of plasma miR-208a and miR-370 expression levels for early diagnosis of coronary artery disease," Biomedical Reports, vol. 5, no. 3, pp. 332-336, 2016.
[41] R. Bellomo, J. A. Kellum, and C. Ronco, "Acute kidney injury," Lancet, vol. 380, no. 9843, pp. 756-766, 2012.

[42] The ad-hoc working group of ERBP, D. Fliser, M. Laville et al., "A European Renal Best Practice (ERBP) position statement on the Kidney Disease Improving Global Outcomes (KDIGO) clinical practice guidelines on acute kidney injury: part 1: definitions, conservative management and contrast-induced nephropathy," Nephrology, Dialysis, Transplantation, vol. 27, no. 12, pp. 4263-4272, 2012.

[43] L. Gu, Y. Tao, C. Chen, Y. Ye, X. Xiong, and Y. Sun, "Initiation of the inflammatory response after renal ischemia/reperfusion injury during renal transplantation," International Urology and Nephrology, vol. 50, no. 11, pp. 2027-2035, 2018.

[44] J. Wang, P. Zhu, R. Li, J. Ren, and H. Zhou, "Fundc1-dependent mitophagy is obligatory to ischemic preconditioningconferred renoprotection in ischemic AKI via suppression of Drp1-mediated mitochondrial fission," Redox Biology, vol. 30, article 101415, 2020.

[45] J. F. Wang, Y. Zha, H. W. Li et al., "Screening plasma miRNAs as biomarkers for renal ischemia-reperfusion injury in rats," Medical Science Monitor, vol. 20, pp. 283-289, 2014.

[46] Y. F. Zou, W. T. Liao, Z. J. Fu, Q. Zhao, Y. X. Chen, and W. Zhang, "MicroRNA-30c-5p ameliorates hypoxiareoxygenation-induced tubular epithelial cell injury via HIF1 $\alpha$ stabilization by targeting SOCS3," Oncotarget, vol. 8, no. 54, pp. 92801-92814, 2017.

[47] E. Belaidi, A. Thomas, G. Bourdier et al., "Endoplasmic reticulum stress as a novel inducer of hypoxia inducible factor-1 activity: its role in the susceptibility to myocardial ischemiareperfusion induced by chronic intermittent hypoxia," International Journal of Cardiology, vol. 210, pp. 45-53, 2016.

[48] Y. Lin, Y. Tang, and F. Wang, "The protective effect of HIF-1 $\alpha$ in T lymphocytes on cardiac damage in diabetic mice," Annals of Clinical and Laboratory Science, vol. 46, no. 1, pp. 32-43, 2016.

[49] H. S. Jung, J. D. Joo, D. W. Kim et al., "Effect of milrinone on the inflammatory response and NF-kB activation in renal ischemia-reperfusion injury in mice," Korean Journal of Anesthesiology, vol. 66, no. 2, pp. 136-142, 2014.

[50] F. L. Sung, T. Y. Zhu, K. K. W. Au-Yeung, Y. L. Siow, and O. Karmin, "Enhanced MCP-1 expression during ischemia/ reperfusion injury is mediated by oxidative stress and NF$\kappa \mathrm{B}$," Kidney International, vol. 62, no. 4, pp. 1160-1170, 2002.

[51] S. Qiu, X. Chen, Y. Pang, and Z. Zhang, "Lipocalin-2 protects against renal ischemia/reperfusion injury in mice through autophagy activation mediated by HIF $1 \alpha$ and NF- $\kappa$ b crosstalk," Biomedicine \& Pharmacotherapy, vol. 108, pp. 244-253, 2018.

[52] I. H. Mallick, W. Yang, M. C. Winslet, and A. M. Seifalian, "Ischemia-reperfusion injury of the intestine and protective strategies against injury," Digestive Diseases and Sciences, vol. 49, no. 9, pp. 1359-1377, 2004.

[53] B. Szebeni, G. Veres, A. Dezsõfi et al., "Increased expression of toll-like receptor (TLR) 2 and TLR4 in the colonic mucosa of children with inflammatory bowel disease," Clinical and Experimental Immunology, vol. 151, no. 1, pp. 34-41, 2008.

[54] J. S. Ainscough, G. Frank Gerberick, M. Zahedi-Nejad et al., "Dendritic Cell IL- $1 \alpha$ and IL- $1 \beta$ Are Polyubiquitinated and Degraded by the Proteasome," Journal of Biological Chemistry, vol. 289, no. 51, pp. 35582-35592, 2014.

[55] C. Chassin, M. Kocur, J. Pott et al., "miR-146a mediates protective innate immune tolerance in the neonate intestine," Cell Host \& Microbe, vol. 8, no. 4, pp. 358-368, 2010. 
[56] K. D. Taganov, M. P. Boldin, K. J. Chang, and D. Baltimore, "NF-kappaB-dependent induction of microRNA miR-146, an inhibitor targeted to signaling proteins of innate immune responses," Proceedings of the National Academy of Sciences of the United States of America, vol. 103, no. 33, pp. 1248112486, 2006.

[57] C. Chassin, C. Hempel, S. Stockinger et al., "MicroRNA-146amediated downregulation of IRAK1 protects mouse and human small intestine against ischemia/reperfusion injury," $E M B O$ Molecular Medicine, vol. 4, no. 12, pp. 1308-1319, 2012.

[58] X. He, Y. Zheng, S. Liu et al., "MiR-146a protects small intestine against ischemia/reperfusion injury by down-regulating TLR4/TRAF6/NF- $\kappa$ B pathway," Journal of Cellular Physiology, vol. 233, no. 3, pp. 2476-2488, 2018.

[59] Y. Han, X. Liao, Z. Gao et al., "Cardiac troponin I exacerbates myocardial ischaemia/reperfusion injury by inducing the adhesion of monocytes to vascular endothelial cells via a TLR4/NF- $\kappa$ B-dependent pathway," Clinical Science (London, England), vol. 130, no. 24, pp. 2279-2293, 2016.

[60] T. M. Scarabelli, A. Stephanou, E. Pasini et al., "Different signaling pathways induce apoptosis in endothelial cells and cardiac myocytes during ischemia/reperfusion injury," Circulation Research, vol. 90, no. 6, pp. 745-748, 2002.

[61] J. Viereck and T. Thum, "Circulating noncoding RNAs as biomarkers of cardiovascular disease and injury," Circulation Research, vol. 120, no. 2, pp. 381-399, 2017.

[62] S. S. Sørensen, A. B. Nygaard, M. Y. Nielsen, K. Jensen, and T. Christensen, "miRNA expression profiles in cerebrospinal fluid and blood of patients with acute ischemic stroke," Translational Stroke Research, vol. 5, no. 6, pp. 711-718, 2014.

[63] S. Xue, W. Zhu, D. Liu et al., "Circulating miR-26a-1, miR146a and miR-199a-1 are potential candidate biomarkers for acute myocardial infarction," Molecular Medicine, vol. 25, no. 1, p. 18, 2019.

[64] F. Wang, G. Long, C. Zhao et al., "Atherosclerosis-related circulating miRNAs as novel and sensitive predictors for acute myocardial infarction," PLoS One, vol. 9, no. 9, article e105734, 2014.

[65] B. Rizzacasa, E. Morini, R. Mango et al., "MiR-423 is differentially expressed in patients with stable and unstable coronary artery disease: a pilot study," PLoS One, vol. 14, no. 5, article e0216363, 2019.

[66] C. Schulte, S. Molz, S. Appelbaum et al., "miRNA-197 and miRNA-223 predict cardiovascular death in a cohort of patients with symptomatic coronary artery disease," PLoS One, vol. 10, no. 12, article e0145930, 2015.

[67] G. C. Jickling, B. P. Ander, X. Zhan, D. Noblett, B. Stamova, and D. Liu, "microRNA expression in peripheral blood cells following acute ischemic stroke and their predicted gene targets," PLoS One, vol. 9, no. 6, article e99283, 2014.

[68] J. Zhou, L. Chen, B. Chen et al., "Increased serum exosomal miR-134 expression in the acute ischemic stroke patients," BMC Neurology, vol. 18, no. 1, p. 198, 2018.

[69] L. Zhang, Y. Xu, S. Xue et al., "Implications of dynamic changes in miR-192 expression in ischemic acute kidney injury," International Urology and Nephrology, vol. 49, no. 3, pp. 541-550, 2017.

[70] S. Samanta, S. Balasubramanian, S. Rajasingh et al., "MicroRNA: a new therapeutic strategy for cardiovascular diseases," Trends in Cardiovascular Medicine, vol. 26, no. 5, pp. 407419, 2016.
[71] Y. B. Ouyang, C. Stary, G. Y. Yang, and R. Giffard, "microRNAs: innovative targets for cerebral ischemia and stroke," Current Drug Targets, vol. 14, no. 1, pp. 90-101, 2013.

[72] T. X. Lu and M. E. Rothenberg, "MicroRNA," The Journal of Allergy and Clinical Immunology, vol. 141, no. 4, pp. 12021207, 2018.

[73] J. F. Lima, L. Cerqueira, C. Figueiredo, C. Oliveira, and N. F. Azevedo, "Anti-miRNA oligonucleotides: a comprehensive guide for design," RNA Biology, vol. 15, no. 3, pp. 338-352, 2018.

[74] Y. Tu, L. Wan, Y. Fan et al., "Ischemic postconditioningmediated miRNA-21 protects against cardiac ischemia/reperfusion injury via PTEN/Akt pathway," PLoS One, vol. 8, no. 10, article e75872, 2013.

[75] Y. Shen, Z. Shen, L. Miao et al., "miRNA-30 family inhibition protects against cardiac ischemic injury by regulating cystathionine- $\gamma$-lyase expression," Antioxidants \& Redox Signaling, vol. 22, no. 3, pp. 224-240, 2015.

[76] K. L. Kozielski, S. Y. Tzeng, and J. J. Green, "siRNA nanomedicine: the promise of bioreducible materials," Expert Review of Medical Devices, vol. 10, no. 1, pp. 7-10, 2013.

[77] G. J. Hannon, "RNA interference," Nature, vol. 418, no. 6894, pp. 244-251, 2002.

[78] D. Peer, "Induction of therapeutic gene silencing in leukocyteimplicated diseases by targeted and stabilized nanoparticles: a mini-review," Journal of Controlled Release, vol. 148, no. 1, pp. 63-68, 2010.

[79] N. Manjunath and D. M. Dykxhoorn, "Advances in synthetic siRNA delivery," Discovery Medicine, vol. 9, no. 48, pp. 418430, 2010.

[80] Z. K. Wang, F. F. Liu, Y. Wang, X. M. Jiang, and X. F. Yu, "Let7 a gene knockdown protects against cerebral ischemia/reperfusion injury," Neural Regeneration Research, vol. 11, no. 2, pp. 262-269, 2016.

[81] K. Wang, F. Liu, C. Y. Liu et al., "The long noncoding RNA NRF regulates programmed necrosis and myocardial injury during ischemia and reperfusion by targeting miR-873," Cell Death and Differentiation, vol. 23, no. 8, pp. 1394-1405, 2016.

[82] J. Zhang, L. Liu, T. Xu et al., "miRspongeR: an R/Bioconductor package for the identification and analysis of miRNA sponge interaction networks and modules," BMC Bioinformatics, vol. 20, no. 1, p. 235, 2019.

[83] R. O. Bak and J. G. Mikkelsen, "miRNA sponges: soaking up miRNAs for regulation of gene expression," Wiley interdisciplinary reviews: RNA, vol. 5, no. 3, pp. 317-333, 2014.

[84] X. Li, Z. Zeng, Q. Li et al., "Inhibition of microRNA-497 ameliorates anoxia/reoxygenation injury in cardiomyocytes by suppressing cell apoptosis and enhancing autophagy," Oncotarget, vol. 6, no. 22, pp. 18829-18844, 2015.

[85] P. E. Nielsen, M. Egholm, R. H. Berg, and O. Buchardt, "Sequence-selective recognition of DNA by strand displacement with a thymine-substituted polyamide," Science (New York, N.Y.), vol. 254, no. 5037, pp. 1497-1500, 1991.

[86] S. Montazersaheb, M. S. Hejazi, and H. Nozad Charoudeh, "Potential of peptide nucleic acids in future therapeutic applications," Advanced pharmaceutical bulletin, vol. 8, no. 4, pp. 551-563, 2018.

[87] S. Malik, J. Lim, F. J. Slack, D. T. Braddock, and R. Bahal, "Next generation miRNA inhibition using short anti-seed PNAs encapsulated in PLGA nanoparticles," Journal of Controlled Release, vol. 327, pp. 406-419, 2020. 
[88] M. M. Fabani, C. Abreu-Goodger, D. Williams et al., "Efficient inhibition of miR-155 function in vivo by peptide nucleic acids," Nucleic Acids Research, vol. 38, no. 13, pp. 4466-4475, 2010.

[89] C. J. Cheng, R. Bahal, I. A. Babar et al., "MicroRNA silencing for cancer therapy targeted to the tumour microenvironment," Nature, vol. 518, no. 7537, pp. 107-110, 2015.

[90] J. Shen, B. Xie, C. M. Hatara, S. F. Hackett, and P. A. Campochiaro, "Vegf or EphA2 Antisense Polyamide-nucleic acids; Vascular Localization and Suppression of Retinal Neovascularization," Molecular therapy : the journal of the American Society of Gene Therapy, vol. 15, no. 11, pp. 1924-1930, 2007.

[91] M. Qu, J. Pan, L. Wang et al., "MicroRNA-126 regulates angiogenesis and neurogenesis in a mouse model of focal cerebral ischemia," Molecular therapy Nucleic acids, vol. 16, pp. 15$25,2019$.

[92] X. Wang, T. Ha, Y. Hu et al., "MicroRNA-214 protects against hypoxia/reoxygenation induced cell damage and myocardial ischemia/reperfusion injury via suppression of PTEN and Bim1 expression," Oncotarget, vol. 7, no. 52, pp. 8692686936, 2016.

[93] E. E. Sayedahmed, R. Kumari, and S. K. Mittal, "Current use of adenovirus vectors and their production methods," Methods in Molecular Biology (Clifton, NJ), vol. 1937, pp. 155-175, 2019.

[94] A. Selvamani and F. Sohrabji, "Mir363-3p improves ischemic stroke outcomes in female but not male rats," Neurochemistry International, vol. 107, pp. 168-181, 2017.

[95] Y. Zhou, Q. Chen, K. S. Lew, A. M. Richards, and P. Wang, "Discovery of potential therapeutic miRNA targets in cardiac ischemia-reperfusion injury," Journal of Cardiovascular Pharmacology and Therapeutics, vol. 21, no. 3, pp. 296-309, 2016. 